\title{
Relações entre perceção da parentalidade, resiliência e rendimento académico no ensino profissional
}

\section{Relationship between parenting perception, resilience and academic performance in vocational education}

\author{
Maria da Luz Vale-Dias, Ana Maia \\ Universidade de Coimbra
}

\begin{abstract}
Resumo
Nesta pesquisa pretendeu-se analisar as relações entre a perceção da qualidade da parentalidade, a resiliência e o rendimento escolar de adolescentes do ensino profissional. Investigaram-se ainda os contributos de variáveis sociodemográficas relevantes. A amostra utilizada incluiu cerca de três centenas de adolescentes a frequentar escolas profissionais no Centro de Portugal, os quais responderam a instrumentos de autorrelato. Para além da importância do papel desempenhado por certas variáveis sociodemográficas, os resultados obtidos sugerem que a perceção do amor parental (versus hostilidade) se relaciona positivamente com os recursos de resiliência, podendo assumir um papel positivo no desenvolvimento adaptativo do adolescente.

Palavras-chave: perceção da parentalidade, adolescentes, resiliência, rendimento académico.
\end{abstract}

\begin{abstract}
The purpose of this research was to analyze the relationship between the perception of parenting quality, resilience and school performance of adolescents in vocational/professional education. The contributions of relevant sociodemographic variables were also analyzed. The sample used in this study included about three hundred adolescents attending professional schools in the Center of Portugal, who responded to self-report instruments. In addition to the importance of the role played by certain sociodemographic variables, the results suggest that the perception of parental love (versus hostility) is positively related to resilience resources, and may have a positive role on adolescent development.

Keywords: parenting perception, adolescents, resilience, academic performance.
\end{abstract}

A família tem sido considerada a primeira mediadora entre o homem e a cultura, surgindo como uma pedra basilar para o desenvolvimento dos seus membros. Apesar de todas as transformações que tem sofrido ao longo do tempo e das sociedades, assumindo a sua polissemia e multivariedade, até ao presente, nenhuma sociedade consegue passar sem a família (Leandro, 2006), ainda que esta adote e evolua para diferentes formas (Relvas, \& Alarcão, 2002). Enquanto contexto de socialização, ela constitui a unidade dinâmica das relações de cunho afetivo, social e cognitivo, assumindo um papel decisivo e essencial no desenvolvimento dos seus elementos (Morgado, Vale Dias, \& Paixão, 2013; Parke, \& Buriel, 2008; Soares, \& Almeida, 2011). Considerada a base crucial da identidade, a família determina as primeiras relações sociais, assim como os contextos onde ocorrem a maior parte das aprendizagens iniciais que efetuamos acerca das pessoas, situações e capacidades individuais (Fleming, 1988). A atmosfera emocional da família, o modo como os filhos são educados, ensinados e preparados para a vida, as oportunidades e dificuldades que a dinâmica familiar coloca ao desenvolvimento normal são fatores que se encontram presentes desde o nascimento e se prolongam ao longo da adolescência (Haro, 2000).

A qualidade das práticas parentais na infância constitui um ponto fulcral no desenvolvimento equilibrado e ajustado da criança. Os pais serão, em princípio, os seus primeiros educadores e agentes socializadores, motivo pelo qual as suas práticas irão, inevitavelmente, influenciar as aprendizagens e o comportamento dos seus filhos no futuro. $\mathrm{O}$ relacionamento familiar, a influência dos estilos parentais e da comunicação familiar desempenham funções importantes no desenvolvimento das crianças e dos adolescentes, assumindo um papel decisivo no ajustamento e aperfeiçoamento de competências psicossociais e na saúde mental (Morgado, \& Vale Dias, 2012, 2013; Morgado, Vale Dias, \& Paixão, 2013; Vale Dias, \& Reboredo, 2011).

Quando se fala da interação parental e das suas práticas educativas, muito abordadas pela literatura (e.g., Baumrind, 1966, 1997), é possível reconhecer duas dimensões diferentes na interação entre pais e filhos. Assim, "as práticas educativas referem-se às estratégias utilizadas pelos pais para atingir objetivos específicos em diferentes domínios (académico, social, afetivo) sob determinadas circunstâncias e contextos [...]. O estilo parental refere-se ao padrão global de características da interação dos pais com os filhos em diversas situações, que geram um clima emocional (Darling \& Steinberg, 1993)" (Cecconello, Antoni, \& Koller, 2003, p.46), podendo o comportamento parental traduzir sobretudo expressões de afetividade, 
responsividade e autoridade, que se designam estilos parentais. Relativamente às práticas parentais, estas alternam entre situações, porém, no que respeita aos estilos parentais, estes parecem coerentes, independentemente da situação e das interações entre pais-crianças (Oliveira et al., 2002; Pacheco, Silveira, \& Schneider, 2008). A análise da tipologia dos estilos parentais, inseparável dos estudos de Baumrind, veio a recolher contributos e reformulações de outros autores (e.g., Darling \& Steinberg, 1993; Maccoby \& Martin, 1983), que ainda hoje inspiram a investigação que pretende esclarecer a qualidade da parentalidade e o seu efeito.

Apesar de a família poder ser considerada normativa, a sua pluralidade e diversidade, tanto no que se refere à sua composição, como à quantidade e qualidade das experiências (López, 1995) é uma realidade. Assim, continuam a justificar-se pesquisas que contribuam para o esclarecimento dos tipos e dimensões da interação pais-filhos, assumida que está a sua importância em diversos campos. Vários estudos têm demonstrado que a qualidade da relação familiar tem um forte impacto em diversas dimensões da vida dos filhos, havendo dados portugueses e internacionais que, na fase da adolescência, mostram a relação com o ajustamento académico (e.g., Soares, \& Almeida, 2011; Lamb, 2010). Ainda, através de uma revisão da literatura acerca da relação entre os estilos de parentalidade e o desempenho escolar dos filhos, Kordi (2010) indicou que existe uma forte associação entre estas variáveis, sendo que o nível educacional e o envolvimento dos pais se encontram relacionados. Além disso, o estilo parental autoritativo, por contraste com o autoritário e o permissivo, de acordo para a tipologia de Baumrind (1966), foi associado a níveis de realização escolar mais elevados. Registaram-se, no entanto, algumas inconsistências, nomeadamente resultados contraditórios em diferentes culturas. Este aspeto demonstra que a investigação em torno desta relação deve ser diversificada. No presente estudo, pretende-se precisamente contribuir neste sentido. Assim, analisaremos a relação entre atitudes parentais e rendimento escolar dos filhos, envolvendo na investigação sujeitos de nacionalidades diferentes, para além de considerar outras variáveis sociodemográficas.

Uma relação de qualidade com os pais também surge como um fator protetor em relação a problemas de comportamento antissocial na adolescência (Vale Dias, \& Reboredo, 2011), promovendo trajetórias socialmente ajustadas no final da infância e na adolescência (Morgado \& Vale-Dias, 2012). Além disso, é a perceção do afeto parental, das relações afetivas entre pais e filhos, o sentimento de possuir uma base segura (ligada às primeiras experiências de vinculação), assim como o sentimento de pertença a uma família, de ser importante e amado pelos pais, que fornece uma segurança interna e favorece a construção de recursos de resiliência (Anaut, 2005, Kaplan, 1999; Rutter, 1999; Werner, 2005). De facto, a resiliência é um processo desenvolvimental dinâmico (Benard, 1991, 1995), que pode ser visto como a capacidade de superar as adversidades da vida e encontrar maneiras de prosperar.
Os pais parecem ser de suma importância na construção da resiliência dos seus filhos, ao cuidar e dar a oportunidade de estabelecerem relacionamentos com modelos positivos. Esta variável será, então, incluída neste estudo.

Partindo da literatura anterior, esta investigação, de caráter exploratório e correlacional, pretendeu contribuir para a análise das relações entre a qualidade da parentalidade, a resiliência e o rendimento escolar de adolescentes do ensino profissional, numa amostra com sujeitos de três nacionalidades diferentes. Para uma melhor compreensão das variáveis em estudo, pretendeu-se, ainda, analisar os eventuais contributos de variáveis demográficas consideradas relevantes pela literatura, tais como a nacionalidade, género, habilitações dos pais, nível socioeconómico e tipo de família. Tendo em conta a revisão de estudos efetuada e o objetivo geral delineado, colocou-se a hipótese de uma relação entre o rendimento escolar, de um lado, e a perceção das atitudes parentais ou a resiliência, de outro. Assim, considerou-se que existe uma relação entre a perceção que o adolescente tem do afeto parental e das atitudes educativas parentais e o seu desempenho académico, associando-se a perceção positiva da qualidade parental a um melhor rendimento académico. Considerou-se, também, existir uma associação positiva entre o desempenho académico e os recursos de resiliência. Colocou-se, ainda, a hipótese da existência de relações entre os vários aspetos dos recursos de resiliência (internos e externos) relatados pelos adolescentes e a perceção que têm do afeto parental e das práticas educativas parentais. Espera-se também a influência das variáveis demográficas a nível das variáveis em estudo (qualidade da parentalidade, resiliência e rendimento escolar), podendo encontrar-se diferenças em função da nacionalidade, do género, do tipo de família, do nível socioeconómico e das habilitações dos pais.

\section{Método}

\section{Participantes}

Este estudo contou com a participação de 282 alunos da Associação Diogo de Azambuja (71.63\% da Escola Profissional de Montemor-o-Velho e $28.37 \%$ da Escola Profissional Agrícola Afonso Duarte), no Centro de Portugal, dos cursos profissionais de nível IV e dos Cursos de Educação e Formação (CEF's) de nível II (do $7^{\circ}$ ao $12^{\circ}$ ano de escolaridade). A Tabela 1 apresenta a distribuição da amostra por escola, nacionalidade, género e local de residência.

É uma amostra maioritariamente do género masculino $(61,70 \%$ de rapazes e $38,30 \%$ de raparigas), a viver sobretudo em áreas rurais $(88.30 \%)$. Os sujeitos apresentam idades que variam entre os 14 e os 23 anos, com uma média de $17.84(\mathrm{DP}=2.02)$, pertencendo a três nacionalidades diferentes: $82.27 \%$ Portugueses, $12.77 \%$ Cabo-verdianos e $4.96 \%$ Santomenses. De acordo com a Classificação Nacional das Profissões, o grupo Operários, Artífices e Trabalhadores Similares foi o mais frequente entre os pais $(35.15 \%)$ e o grupo Trabalhadores Não Qualificados o mais frequente entre 
as mães (67.31\%). As habilitações literárias dos progenitores que predominam são as habilitações entre o $1^{\circ}$ e $3^{\circ}$ ciclos, tanto entre os pais $(84.31 \%)$ como entre as mães $(82.20 \%)$. Relativamente à situação face ao emprego, $90.04 \%$ das mães e $82.31 \%$ dos pais estão empregados e o nível socioeconómico (NSE) baixo é o mais frequente $(75.89 \%)$, tendo respondido ao questionário apenas três sujeitos classificados no NSE alto.

Tabela1.

Distribuição da amostra por escola, nacionalidade, género e local de residência

\begin{tabular}{llcc}
\hline & & $\mathrm{n}$ & $\%$ \\
\hline Escola & E. P. de Montemor & 202 & 71.63 \\
& E. P. Agrícola & 80 & 28.37 \\
Nacionalidade & Portuguesa & 232 & 82.27 \\
& Cabo-verdiana & 36 & 12.77 \\
& Santomense & 14 & 4.96 \\
Género & Masculino & 174 & 61.70 \\
& Feminino & 108 & 38.30 \\
Local & & & \\
de residência & Urbano & 33 & 11.70 \\
\hline
\end{tabular}

\section{Instrumentos}

Após acautelados todos os procedimentos éticos, incluindo as necessárias autorizações e consentimento informado, a recolha de dados foi efetuada, junto dos adolescentes, através da aplicação de um questionário sociodemográfico e, ainda, de mais dois instrumentos e de uma medida do rendimento escolar. Este foi avaliado através das médias das classificações finais (ano 2011-2012) de cada um dos três níveis da estrutura modular de ensino Profissional (sociocultural, cientifico e técnico, e tecnológico dos cursos de nível II e nível IV).

A qualidade da parentalidade foi medida a partir da intensidade da perceção dos adolescentes acerca das atitudes parentais (dimensão Afeto: amor/hostilidade; e dimensão Práticas parentais: autonomia/controlo), através do Inventário de Perceções Adolescentes - IPA (Streit, 1978; versão port: Fleming, 1997). A resiliência foi avaliada através do California Healthy Kids Survey Resilience Assessment Module /Versao 6.0 - HKRA (Constantine \& Benard 2001; versão port: Martins, 2005), considerando os recursos de resiliência (internos e externos) percecionados pelo adolescente. Os instrumentos apresentaram as adequadas propriedades psicométricas.

\section{Resultados}

Os dados revelam interessantes contributos das variáveis demográficas a nível das variáveis em estudo, assim como relações entre as atitudes parentais e a resiliência dos adolescentes.

\section{Nacionalidade e Inventário de Percepções Adolescentes (IPA)}

Foram comparados os scores das subscalas do IPA de dois grupos de sujeitos, segundo a nacionalidade, isto é, Portugueses $(\mathrm{N}=232)$ e Africanos (Cabo-verdianos e Santomenses, $\mathrm{N}=50$ ). Através de uma ANOVA com correção de Brown-Forsythe, os resultados revelam diferenças em função da nacionalidade. Na dimensão Afeto - IPA, a perceção de Hostilidade é estatisticamente superior nos alunos africanos $(\mathrm{F}=$ 6.012; $p=0.15)$ e, na dimensão das Práticas educativas, a perceção da Autonomia parental é estatisticamente superior nos alunos portugueses $(\mathrm{F}=23.174 ; p \leq .001)$. Este dado sugere que os adolescentes Africanos percecionam a qualidade do afeto parental como mais hostil e também sentem menos autonomia parental.

\section{Nacionalidade e Resiliência (HKRA)}

Relativamente ao HKRA, após uma ANOVA, verificou-se que os alunos portugueses apresentam resultados estatisticamente superiores na subescala recursos externos - Envolvimento na escola $(\mathrm{F}=5.750$; $p=0.19)$ e na escala recursos internos $(\mathrm{F}=18.270 ; p \leq$ .001 ), sugerindo que os portugueses apresentam mais recursos nestas dimensões que os africanos. Ou seja, os portugueses parecem percecionar-se de forma mais positiva em relação às suas competências sociais, autonomia, objetivos de vida (ao nível dos recursos internos) e quanto ao envolvimento em atividades relevantes, com oportunidades para a responsabilidade e contributo pessoal (ao nível dos recursos externos Envolvimento na escola).

\section{Género e Inventário de Percepções Adolescentes (IPA)}

Os rapazes tendem a apresentar valores de Perceção de Autonomia $(\mathrm{F}=10.729 ; p=.001)$ e de Controlo parental $(\mathrm{F}=4.855 ; p=.028)$ significativamente superiores aos das raparigas.

\section{Género, Resiliência (HKRA) e Resultados Escolares}

As raparigas revelam médias estatisticamente superiores na escala Recursos Internos, indicando que as elas apresentam mais recursos nesta dimensão $(\mathrm{F}=9.608 ; p=.002)$. Elas mostram também melhores resultados escolares que os colegas do género masculino $(\mathrm{F}=10.701 ; p=.001)$.

\section{Tipo de família, IPA e HKRA}

Foram considerados três tipos de família: as Intactas (tradicionais), as Monoparentais e as Reconstituídas. Após a realização de ANOVA e testes post hoc, as famílias intactas apresentaram uma média estatisticamente superior aos restantes tipos de famílias na escala de Perceção de Amor $(\mathrm{F}=8.901 ; p \leq .001)$, Perceção de Controlo $(\mathrm{F}=18.256 ; p \leq .001)$ e Perceção de Autonomia $(\mathrm{F}=14.079 ; p \leq .001)$, sugerindo que os adolescentes oriundos de famílias intactas apresentam uma perceção da relação com os pais mais satisfatória do que os adolescentes que vivem noutros tipos de estruturas familiares. No que se refere à resiliência (HKRA), uma ANOVA revelou diferenças 
sigfnificativas entre os três tipos de família, mas os testes post hoc foram inconclusivos na identificação dos grupos que diferiam entre si.

\section{Habilitações literárias do pai e da mãe, IPA, HKRA e resultados escolares}

Consideraram-se três grupos de habilitações: a) até ao $1^{\circ}$ ciclo; b) $2^{\circ}$ e $3^{\circ}$ ciclos; c) secundário e ensino superior. Analisados os resultados das ANOVAs, verificou-se que as habilitações literárias da mãe não mostraram efeito significativo em nenhuma das VDs. Quanto ao pai, as análises de variância revelaram diferenças significativas na escala Hostilidade $(F(2,157.04)=3.152, p=.045)$, verificando-se a média mais alta no grupo do secundário e ensino superior. Relativamente à resiliência, houve diferenças na escala recursos externos $(F(2,203.54)=5.216, p=.006)$, na escala Envolvimento na comunidade $(F(2,187.08)=$ $3.797, p=.024)$ e no Envolvimento no grupo de pares $(F(2,171.28)=5.188, p=.006)$, sendo os filhos de pais com habilitações até ao $1^{\circ}$ ciclo que obtêm uma média significativamente inferior à que foi obtida pelos filhos de pais com graus superiores ao seu. Também é neste grupo de pais, menos qualificados, que se registam os resultados escolares significativamente mais baixos $(p=.010)$, obtidos pelos filhos.

\section{Estudo de correlações (Pearson)}

Embora não tenham sido encontradas correlações relevantes, quer pelo nível de significância, quer pela sua força, entre as variáveis resultados escolares, de um lado, e as atitudes parentais ou a resiliência, do outro, os dados revelam algumas relações interessantes entre as atitudes parentais e a resiliência. $\mathrm{Na}$ dimensão Afeto Parental-IPA, a escala Perceção do Amor associa-se positivamente às escalas Recursos Externos ( $\mathrm{r}=.339)$, Envolvimento em casa ( $\mathrm{r}=.475)$, Envolvimento na comunidade $(\mathrm{r}=.226)$ e Envolvimento no grupo de pares $(\mathrm{r}=.241)$ do HKRA. Já a Perceção de Hostilidade-IPA aparece negativamente associada à escala de Recursos Externos ( $\mathrm{r}=-, 302)$, ao Envolvimento em casa $(\mathrm{r}=-, 261)$, ao Envolvimento na comunidade $(\mathrm{r}=-, 291)$ e ao Envolvimento no grupo de pares $(\mathrm{r}=-, 297)$. Estes resultados são estatisticamente significativos $(p<.001) \mathrm{e}$ vão de encontro ao esperado.

\section{Discussão e Conclusão}

Considerando a relevância da investigação das atitudes parentais e assumindo que o comportamento e desenvolvimento do ser humano são multideterminados, resultado da influência de múltiplas variáveis, esta pesquisa debruçou-se sobre as relações entre a perceção das atitudes parentais, a resiliência e o rendimento escolar, examinando, também, a influência de variáveis sociodemográficas relevantes. Apesar da ausência, também assinalada noutros estudos, de associações significativas entre o rendimento escolar, de um lado, e as atitudes parentais ou a resiliência, de outro, os dados permitem destacar algumas correlações de interesse entre a perceção das atitudes parentais e a avaliação da resiliência. Com efeito, os resultados sugerem, de um modo geral, que a perceção positiva do afeto parental (amor), pelos adolescentes, se relaciona com os seus recursos de resiliência, nomeadamente os recursos externos e o envolvimento em casa, na comunidade e no grupo de pares, podendo ter um papel positivo no desenvolvimento adaptativo do adolescente em diversas áreas (social, académica, da saúde mental, etc.) e vice-versa. No entanto, face aos dados, um raciocínio similar deve ser feito relativamente à perceção da hostilidade, refletindo sobre possíveis associações inversas e nefastas para a resiliência. Considerando a sua relação com esta, as atitudes parentais positivas (amor) podem ser conceptualizadas como um recurso externo.

Para Constantine \& Benard (2001), os fatores protetores externos têm um papel importante na promoção de resultados positivos em todos os contextos e ambientes, e embora sejam vistos como externos, estes fatores protetores também são influenciados pelo adolescente (pela sua perceção e pela sua capacidade de os desenvolver). Os traços de resiliência, usualmente vistos como constructos internos, são também, em certa medida, influenciados pelo ambiente externo. Estes autores defendem, ainda, que cada um dos fatores protetores externos irá influenciar o processo do desenvolvimento psicossocial do adolescente, manifestando-se essa influência a nível dos traços internos de resiliência, conduzindo assim a resultados desenvolvimentais nas áreas social, académica e da saúde.

De acordo com o esperado, os dados revelaram contributos interessantes das variáveis sociodemográficas nos constructos em estudo, tendo surgido diferenças em função da nacionalidade, género, tipo de família e habilitações paternas. Tais resultados merecem futuro aprofundamento, nomeadamente no que respeita à nacionalidade e às habilitações do pai. Questões mais aprofundadas relativas à cultura e ao papel do pai deverão ser equacionadas. Por exemplo, há que continuar a refletir sobre as diferenças culturais em torno das atitudes parentais, tal como outros estudos vêm fazendo. Quanto ao resultado referente ao pai, ele parece ser consistente com alguma literatura que aponta para um maior envolvimento do pai na educação e desenvolvimento dos filhos (Hall, 2005; Lamb, 2010).

A presente pesquisa trouxe alguns contributos interessantes, permitindo também refletir sobre dados de uma investigação que incluiu indivíduos de um tipo de ensino específico, com alguma diversidade cultural e pertencentes maioritariamente a meio desfavorecido. Certos resultados permitirão a elaboração de propostas no que concerne a estratégias de prevenção/intervenção. Algumas limitações devem ser também mencionadas. O facto de terem sido utilizadas medidas de autorresposta pode enviesar os resultados. Será desejável incluir, no futuro, outro tipo de instrumentos e mais fontes de dados, tais como os próprios pais. Algumas variáveis familiares, por exemplo, a organização da família, sua qualidade relacional e história, deverão ser consideradas. O mesmo se pode referir em relação ao controlo de fatores pessoais (por ex., personalidade). Finalmente assinale-se a importância de trabalhar com 
amostras mais equilibradas e de desenvolver estudos longitudinais.

\section{Referências}

Anaut, M. (2005). A Resiliencia - Ultrapassar os Traumatismos. Lisboa: Climepsi Editores.

Baumrind, D. (1966). Effects of authoritative parental control on child behavior. Child Development, 37, 887-907. DOI: $10.2307 / 1126611$

Baumrind, D. (1997). The discipline encounter: contemporary issues. Aggression and Violent Behavior, 2, 321-335. doi.org/10.1016/S1359-1789(97)00018-9

Benard, B. (1991). Protective Factors in the Family, School, and Community. Portland, OR: Western Center for Drug-Free Schools and Communities.

Benard, B. (1995). Fostering resilience in children. Champaign,IL: ERIC Clearinghouse on Elementary and Childhood Education.

Bornstein, M. (Ed.) (2002). Handbook of Parenting. Mahwah, NJ: Erlbaum.

Cecconello, A. M. Antoni, C. \& Koller, S. H. (2003). Práticas educativas, estilos parentais e abuso físico no contexto familiar. Psicologia em Estudo, 8, 45-54. http://www.scielo.br/pdf/pe/v8nspe/v8nesa07

Constantine, N.A. \& Benard, B. (2001). California Healthy Kids Survey Resilience Assessment Module: Technical Report. Berkeley, CA: Public Health Institute.

Darling, N. \& Steinberg, L. (1993). Parenting style as context: An integrative model. Psychologícal Bulletín, 113 , 487 -496. doi:10.1037/0033-2909.113.3.487

Fleming, M. (1988). Autonomia comportamental na adolescência e perceções das atitudes parentais. Tese de doutoramento apresentada ao Instituto de Ciências Biomédicas de Abel Salazar: Universidade do Porto.

Fleming, M. (1997). Adolescência e autonomia. O desenvolvimento psicológico e a relação com os pais. $2^{\circ}$ ed.Porto: Edições Afrontamento.

Hall, S. (2005). Change in paternal involvement from 1977 to 1997: A cohort analysis. Family and Consumer Sciences Research Journal, 34, 127-139. DOI: $10.1177 / 1077727 \mathrm{X} 05280664$

Haro, E. F. (2000). Educacíon familiar y desarrollo de los hijos. In A. M. Fontaine (Coord.), Parceria família-escola e desenvolvimento da criança (pp. 19-30). Porto: Edições Asa.

Kaplan, H. B. (1999). Toward an understanding of resilience: A critical review of definitions and models. In M. D. Glantz \& J. L. Johnson (Orgs.), Resilience and development. Positive life adaptations (pp. 17-83). New York: Plenum Press.

Kordi, A. (2010). Parenting Attitude and Style and Its Effect on Children's School Achievements. International Journal of Psychological Studies, 2 (2), 217-222. doi: 10.1080/03004430.2015.1083559

Lamb, M. (Ed.). (2010). The role of the father in child development (5th ed.). Hoboken, NJ: Wiley.

Leandro, M. E. (2006). Transformações da família na história do Ocidente. Theologica, 2. ${ }^{\mathrm{a}}$ Série, 41(1), 51-74.http://repositorio.ucp.pt/bitstream/10400.14/12 875/1/leandro.pdf

López, F. (1995). Necesidades de la infancia: respuesta familiar. Infancia y Sociedade, 30, 7-48.

Maccoby, E. E \& Martin. J. A (1983). Socialization in the context ofthe family: parent-child intenction. In P. H. Hussen (Series Ed.) \& E. M. Hetherington (Vol. Ed). Handbook of Child Psychology. New York: Wiley

Martins, M. H. (2005). Contribuições para a análise de crianças e jovens em situação de risco -Resiliência e Desenvolvimento. Tese de Doutoramento em Psicologia, na área da Psicologia Educacional. Faculdade de Ciências Humanas e Sociais da Universidade do Algarve, Faro, Portugal.

Morgado, A. \& Vale-Dias, M. L. (2012). Promoting socially adjusted trajectories in late childhood and adolescence. INFAD, International Journal of Developmental and Educational Psychology, XXIV, 1, Vol.1, 647-656. http://dehesa.unex.es/handle/10662/3574?show=full

Morgado, A. M., \& Vale-Dias, M. L. (2013). The antisocial phenomenon in adolescence: What is literature telling us? Aggression and Violent Behavior,

18,436-443.http://dx.doi.org/10.1016/j.avb.2013.05.0 04

Morgado, A., Vale-Dias, M. L. \& Paixão, M. P. (2013). O desenvolvimento da socialização e o papel da família. Análise Psicológica, 2 (XXXI), 1-15. http://www.scielo.mec.pt/scielo.php?script=sci arttex t\&pid $=$ S0870-82312013000200002

Oliveira, E., Marin, A., Pires, F., Frizzo, G., Ravanello, T. \& Rossato, C. (2002). Estilos Parentais Autoritário e Democrático-Recíproco Intergeracionais, Conflito Conjugal e Comportamentos de Externalização e Internalização. Psicologia; Reflexão e Crítica, 15 (1), $1-11$.

Pacheco, J., Silveira, L. \& Schneider, A. (2008). Estilos e práticas educativas parentais: análise da relação desses constructos sob a perspetiva dos adolescentes. Psico, 39 (1), 66-73.

Parke, R. D., \& Buriel, R. (2008). Socialization in the family: Ethnic and ecological perspectives. In W. Damon \& R. M. Lerner (Eds.), Child and adolescent development: An advanced course (pp. 95-138). Hoboken, N.J.: Wiley.

Relvas, A. P. \& Alarcão, M. (2002). Novas formas de família. Coimbra: Quarteto.

Rutter, M. (1999) Resilience concepts and findings: implications for family therapy. Journal of Family Therapy, DOI: $10.1111 / 1467-6427.00108$

Soares, D. \& Almeida, L. (2011). Perceção dos Estilos Educativos Parentais: sua Variação ao Longo da Adolescência. Libro de Atas do XI Congresso Internacional Galego- Português de Psicopedagogia, 4071-4083. http://hdl.handle.net/1822/15346

Streit, F. (1978). Technical manual: Youth Perception Inventory. Fred Streit Associates.

Vale Dias, M. L. \& Reboredo, A. R. (2011). L'Influence de la Perception des Relations Parents-Enfants dans le 
Comportement Antisocial. In E. Catarsi et J.-P. Pourtois (Eds.), Education familiale et services pour l'enfance, II vol. (pp. 119-126). Firenze: Firenze University Press.

Werner, E. (2005).What can we learn about resilience from large-scale longitudinal studies. In: Sam Goldstein, \& Robert B. Brooks (Eds.). Handbook of resilience in children (pp. 91-105). New York: Kluwer Academic. Plenum Publishers.

\section{Agradecimientos}

Trabalho realizado no âmbito do Instituto de Psicologia Cognitiva, Desenvolvimento Humano e Social (I\&D), FCT. 